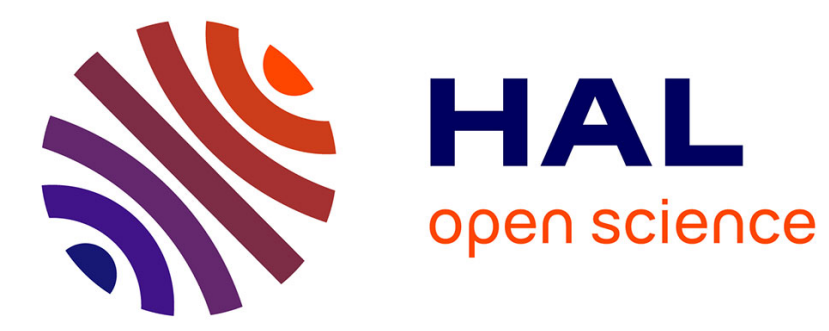

\title{
Quasi-Affine Transformation in 3-D: Theory and Algorithms
}

David Coeurjolly, Valentin Blot, Marie-Andrée Jacob-da Col

\section{To cite this version:}

David Coeurjolly, Valentin Blot, Marie-Andrée Jacob-da Col. Quasi-Affine Transformation in 3-D: Theory and Algorithms. Combinatorial Image Analysis, Nov 2009, Playa del Carmen, Mexico. pp.68 - 81, 10.1007/978-3-642-10210-3_6. hal-01092500

\section{HAL Id: hal-01092500 https://hal.science/hal-01092500}

Submitted on 8 Dec 2014

HAL is a multi-disciplinary open access archive for the deposit and dissemination of scientific research documents, whether they are published or not. The documents may come from teaching and research institutions in France or abroad, or from public or private research centers.
L'archive ouverte pluridisciplinaire HAL, est destinée au dépôt et à la diffusion de documents scientifiques de niveau recherche, publiés ou non, émanant des établissements d'enseignement et de recherche français ou étrangers, des laboratoires publics ou privés. 


\title{
Quasi-Affine Transformation in 3-D: Theory and Algorithms
}

\author{
David Coeurjolly $^{1}$, Valentin Blot ${ }^{2}$ and Marie-Andrée Jacob-Da Col ${ }^{3}$ \\ 1 Université de Lyon, CNRS, LIRIS, UMR5205, F-69622, France \\ david.coeurjolly@liris.cnrs.fr \\ 2 Ecole Normale Supérieure de Lyon valentin.blot@ens-lyon.fr \\ 3 LSIIT-UMR 7005, Pôle API Bd Sébastien Brant, Illkirch, F-67412, France \\ dacolm@iutlpa.u-strasbg.fr
}

\begin{abstract}
In many applications and in many fields, algorithms can considerably be speed up if the underlying arithmetical computations are considered carefully. In this article, we present a theoretical analysis of affine transformations in dimension 3. More precisely, we investigate the arithmetical paving induced by the transformation to design fast algorithms.
\end{abstract}

Key words: quasi-affine transform, periodic tiling, arithmetic, image transformation.

\section{Introduction}

In many computer vision and image processing applications, we are facing new constraints due to the image sizes both in dimension with 3-D and 3-D+t medical acquisition devices, and in resolution with VHR (Very High Resolution) satellite images. This article deals with high performance image transformations using quasi-affine transforms (QATs for short), which can be viewed as a discrete version of general affine transformations. QAT can approximate rotations and scalings, and in some specific cases, QAT may also be one-to-one and onto mappings from $\mathbb{Z}^{n}$ to $\mathbb{Z}^{n}$, leading to exact computations. In dimension 2, the QAT appeared in several articles [1/23/4/5]. In higher dimension, theoretical results have been demonstrated [6/7/8]. More precisely, authors have demonstrated the arithmetical and periodic structures embedded in $n$-dimensional QAT, leading to generic transformation algorithms. To implement these generic algorithms, several elements have to be fixed when considering a specific dimension. In this paper, we detail the computation of the minimal periods in dimension 3 leading to efficient transformation algorithms. Due to the space limitation, the proofs are available in the technical report [7). In Section 2, we first present some definitions and results on $n-\mathrm{D}$ QAT. Section 3 focuses on the parameter computation in 3-D. Finally, Section 4 evaluate all the algorithms compared to a classical backward-mapping technique [9]. 


\section{Preliminaries}

In this section, we present definitions and results obtained for QAT in higher dimension. These results have been independently proved by [6] and $[7 / 8$. In the following, we consider notations introduced in 778. Let $n$ denote the dimension of the considered space, $V_{i}$ the $i^{t h}$ coordinate of vector $V$, and $M_{i, j}$ the $(i, j)^{t h}$ coefficient of matrix $M$. We use the notation $\operatorname{gcd}(a, b, \ldots)$ for the greatest common divisor of an arbitrary number of arguments, and $\operatorname{lcm}(a, b, \ldots)$ for their least common multiple. Let $\left[\frac{a}{b}\right]$ denote the quotient of the euclidean division of $a$ by $b$, that is the integer $q \in \mathbb{Z}$ such that $a=b q+r$ satisfying $0 \leq r<|b|$ regardless of the sign of $y^{4}$ We also consider the straightforward generalization of these operators to $n$-dimensional vectors $\left(e . g .\left[\frac{V}{b}\right]\right.$ is a vector where each component is the quotient of the division by $b$ ).

Definition 1. A quasi-affine transformation is a triple $(\omega, M, \boldsymbol{V}) \in \mathbb{Z} \times M_{n}(\mathbb{Z}) \times$ $\mathbb{Z}^{n}$ (we assume that $\operatorname{det}(M) \neq 0$ ). The associated application is :

$$
\begin{aligned}
\mathbb{Z}^{n} \longrightarrow \mathbb{Z}^{n} & \\
X \longmapsto & {\left[\frac{M X+V}{\omega}\right] }
\end{aligned}
$$

Definition 2. The inverse of a $Q A T(\omega, M, V)$ is the $Q A T$ :

$$
\left(\operatorname{det}(M), \omega \operatorname{com}(M)^{t},-\operatorname{com}(M)^{t} V\right),
$$

where $M^{t}$ denotes the transposed matrix and $\operatorname{com}(M)$ the co-factor matrix of $M$ (Remind that $M \operatorname{com}(M)^{t}=\operatorname{com}(M)^{t} M=\operatorname{det}(M) I_{n}$.).

The associated affine application of the inverse of a QAT is therefore the inverse of the affine application associated to the QAT. However, due to the nested floor function, the composition $f \cdot f^{-1}$ is not the identity function in the general case. Let us recall the well-known Bezout Identity:

$$
\forall(a, b) \in \mathbb{Z}^{2}, \exists(u, v) \in \mathbb{Z}^{2} / a u+b v=\operatorname{gcd}(a, b) .
$$

In Section 3, we have to consider a generalized form of the Bezout identity in dimension 3:

Proposition 1 ([7]). $\forall(a, b, c) \in \mathbb{Z}^{3}, \exists(u, v, w) \in \mathbb{Z}^{3} / a u+b v+c w=\operatorname{gcd}(a, b, c)$.

We present now several results and definitions that have been presented for $n$-dimensional QAT. All these results are given in $[7,8$ but we present here the main theorems, which will be used in the rest of the paper. First, the key feature of $n-\mathrm{D}$ QAT is that it contains a periodic paving structure.

Definition 3 (Tile). Let $f$ be a $Q A T$. For $Y \in \mathbb{Z}^{n}$, we denote:

$$
P_{Y}=\left\{X \in \mathbb{Z}^{n} / f(X)=Y\right\},
$$

$P_{Y}$ is called order 1 tile of index $Y$ of $f$.

\footnotetext{
${ }^{4}\left\{\frac{a}{b}\right\}$ denotes the corresponding remainder $\left\{\frac{a}{b}\right\}=a-b\left[\frac{a}{b}\right]$.
} 


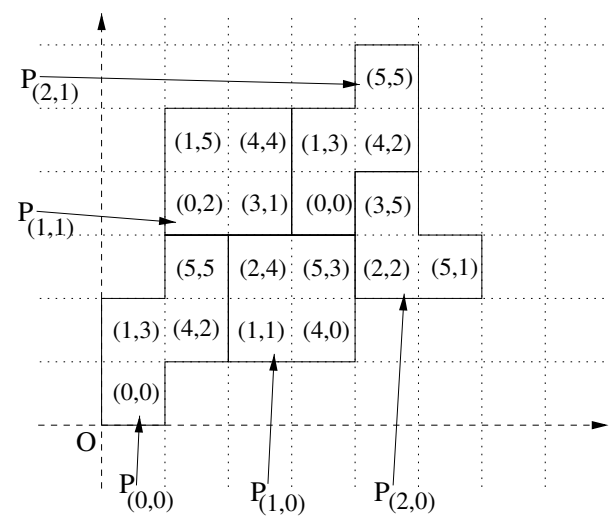

Fig. 1. Example of tiles and remainders

Definition 4. $P_{Y}$ is said arithmetically equivalent to $P_{Z}$ (denoted $P_{Y} \equiv P_{Z}$ ) if:

$$
\forall X \in P_{Y}, \exists X^{\prime} \in P_{Z} /\left\{\frac{M X+V}{\omega}\right\}=\left\{\frac{M X^{\prime}+V}{\omega}\right\} .
$$

Definition 5. $P_{Y}$ and $P_{Z}$ are said geometrically equivalent if:

$$
\exists \boldsymbol{v} \in \mathbb{Z}^{n} / P_{Y}=T_{\boldsymbol{v}} P_{Z},
$$

where $T_{\boldsymbol{v}}$ denotes the translation of vector $\boldsymbol{v}$.

The following theorem exhibits a relation between the notions of arithmetically and geometrically equivalent tiles.

Theorem 1 ([78 $]$ ). If $P_{Y} \equiv P_{Z}$, then $P_{Y}$ and $P_{Z}$ are geometrically equivalent. Since $P_{Y} \equiv P_{Z}$, there exists $X \in P_{Y}$ and $X^{\prime} \in P_{Z}$ such that:

$$
\left\{\frac{M X+V}{\omega}\right\}=\left\{\frac{M X^{\prime}+V}{\omega}\right\} \text {. }
$$

Then $\boldsymbol{v}=X-X^{\prime}$ is the translation vector:

$$
P_{Y}=T_{\boldsymbol{v}} P_{Z}
$$

In Figure 1 we show some tiles of the QAT defined by $\left(6,\left(\begin{array}{rr}3 & 1 \\ -1 & 3\end{array}\right),\left(\begin{array}{l}0 \\ 0\end{array}\right)\right)$ (a point of $\mathbb{Z}^{2}$ is represented by a unit square whose bottom-left corner corresponds to the represented point). For each point $X$ in a tile we provide its corresponding remainder $\left\{\frac{M X+V}{\omega}\right\}$. Tiles $P_{(2,1)}$ and $P_{(0,0)}$ are arithmetically equivalent, therefore they are also geometrically equivalent. It should also be noted that tiles $P_{(1,0)}$ and $P_{(1,1)}$ are geometrically equivalent but they are not arithmetically equivalent . 
Definition 6 (Periodicity notations, [7,8]). For all $0 \leq i<n$, We define the set $\mathcal{A}_{i}$ as follows:

$$
\begin{aligned}
\mathcal{A}_{i}=\left\{\alpha \in \mathbb{N}^{*} / \exists\left(\beta_{j}\right)_{0 \leq j<i} \in \mathbb{Z}^{i}, \forall\left(y_{0}, \ldots, y_{n-1}\right) \in \mathbb{Z}^{n},\right. \\
\left.P_{y_{0}, \ldots, y_{i}+\alpha, \ldots, y_{n-1}} \equiv P_{y_{0}+\beta_{0}, \ldots, y_{i-1}+\beta_{i-1}, y_{i}, \ldots, y_{n-1}}\right\}
\end{aligned}
$$

Furthermore, let us consider $\alpha_{i}=\min \left(\mathcal{A}_{i}\right)$. We define $\left\{\beta_{j}^{i}\right\}_{0 \leq j<i} \in \mathbb{Z}^{i}$ and $U_{i} \in \mathbb{Z}^{n}$ such that

$$
\forall\left(y_{0}, \ldots, y_{n-1}\right) \in \mathbb{Z}^{n}, P_{y_{0}, \ldots, y_{i}+\alpha_{i}, \ldots, y_{n-1}}=T_{\boldsymbol{U}_{i}} P_{y_{0}+\beta_{0}^{i}, \ldots, y_{i-1}+\beta_{i-1}^{i}, y_{i}, \ldots, y_{n-1}} .
$$

The key theorem for the QAT periodic structure can now be presented:

Theorem 2 (Periodicity, [7,8]). The set of QAT tiles is $n$-periodic, in other words

$$
\forall 0 \leq i<n, \mathcal{A}_{i} \neq \emptyset
$$

Let us suppose that quantities $\alpha_{i}, \beta_{j}^{i}$ and $U_{i}$ are given. The following theorem allows us to obtain $P_{Y}$ as the translation of a canonical tile $P_{Y^{0}}$.

Theorem $3([\mathbf{7}, \mathbf{8}]) \cdot \forall\left(y_{0}, \ldots, y_{n-1}\right) \in \mathbb{Z}^{n}$, we have $P_{y_{0}, \ldots, y_{n-1}}=T_{W} P_{y_{0}^{0}, \ldots, y_{n-1}^{0}}$ with

$$
W=\sum_{i=0}^{n-1} w_{i} U_{i} \quad \text { and } \quad \forall n>i \geq 0,\left\{\begin{array}{l}
w_{i}=\left[\frac{y_{i}+\sum_{j=i+1}^{n-1} w_{j} \beta_{i}^{j}}{\alpha_{i}}\right] \\
y_{i}^{0}=\left\{\frac{y_{i}+\sum_{j=i+1}^{n-1} w_{j} \beta_{i}^{j}}{\alpha_{i}}\right\}
\end{array}\right.
$$

In 788 , we have proved that canonical tiles $P_{Y^{0}}$ are associated to grid points of a special tile called super-tile.

Definition 7 (Super-tile, [7,8]). A super-tile of a $Q A T$ is the set $\mathcal{P}$ such that

$$
\mathcal{P}=\bigcup_{0 \leq Y^{0}<\left(\alpha_{0}, \ldots, \alpha_{n-1}\right)} P_{Y^{0}}
$$

Theorem 4 ([7] 8$]) . \mathcal{P}$ is the tile $P_{(0, \ldots, 0)}$ of the $Q A T$ defined by:

$$
\left(\omega \operatorname{lcm}_{0 \leq i<n}\left(\alpha_{i}\right),\left(\begin{array}{ccc}
\theta_{0} & \cdots & 0 \\
\vdots & \ddots & \vdots \\
0 & \cdots & \theta_{n-1}
\end{array}\right) M,\left(\begin{array}{ccc}
\theta_{0} & \cdots & 0 \\
\vdots & \ddots & \vdots \\
0 & \cdots & \theta_{n-1}
\end{array}\right) V\right)
$$

with $\forall 0 \leq i<n-1, \theta_{i}=\frac{\operatorname{lcm}_{0 \leq j<n-1}\left(\alpha_{j}\right)}{\alpha_{i}}$.

Figure 2 illustrates tiles of the QAT $\left(84,\left(\begin{array}{rr}12 & -11 \\ 18 & 36\end{array}\right),\left(\begin{array}{l}0 \\ 0\end{array}\right)\right)$ in $\mathbb{Z}^{2}$ with 15 arithmetically distinct tiles (the tiles with same color are arithmetically equivalent). In this example, for all $i, j \in \mathbb{N}, P_{(i+5, j)} \equiv P_{(i, j)}$ and $P_{(i+2, j-3)} \equiv P_{(i, j)}$. 


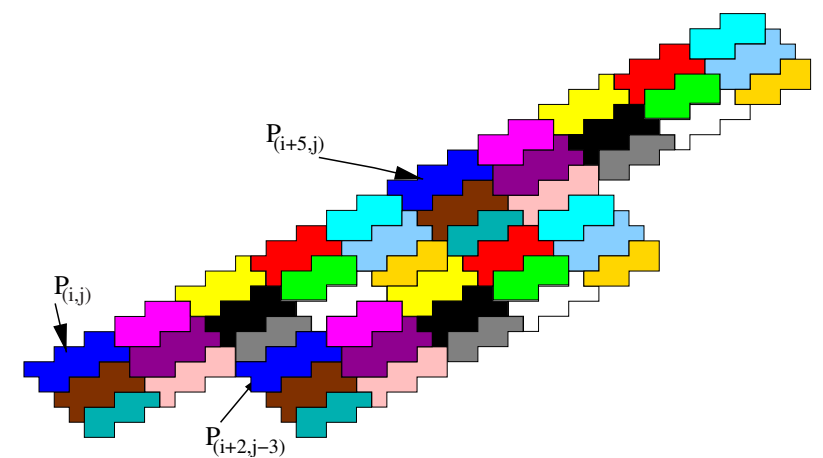

Fig. 2. Periodicity of thes tiles of a 2D QAT .

The set $\left\{P_{(i, j)} \mid i=0,1,2,3,4, j=0,1,2\right\}$ contains exactly once all distinct tiles so it is a super-tile of the QAT.

In 78 , we have demonstrated that if we consider the Hermite Normal Form of the QAT matrix $M$, then efficient tile construction can be designed. In the following, let $M H=T$ denote the Hermite Normal Form (with $\operatorname{det}(H)= \pm 1$ and $T$ upper triangular). Note that this form always exists for nonsingular integer square matrix.

Theorem 5 (Tile Construction, [7,8]). $\forall Y \in \mathbb{Z}^{n}$, let $M H=T$ be the Hermite Normal Form of the QAT matrix $M$,

$$
\begin{gathered}
P_{Y}=\left\{H X / \forall n>i \geq 0, A_{i}\left(X_{i+1}, \ldots, X_{n-1}\right) \leq X_{i}<B_{i}\left(X_{i+1}, \ldots, X_{n-1}\right)\right\} \\
\text { With } A_{i}\left(X_{i+1}, \ldots, X_{n-1}\right)=-\left[\frac{-\omega Y_{i}+\sum_{j=i+1}^{n-1} T_{i, j} X_{j}+V_{i}}{T_{i, i}}\right] \\
B_{i}\left(X_{i+1}, \ldots, X_{n-1}\right)=-\left[\frac{-\omega\left(Y_{i}+1\right)+\sum_{j=i+1}^{n-1} T_{i, j} X_{j}+V_{i}}{T_{i, i}}\right] .
\end{gathered}
$$

In Algorithm 1, we give the generic algorithm applying a contracting QAT $f$ to an image $\mathcal{A}$ (see Fig. 3). The principle is that we give to each pixel $Y$ of image $\mathcal{B}$ the average color of the tile $P_{Y}$ in image $\mathcal{A}$. If $f$ is a dilating QAT, we obtain a similar algorithm in which we replace $f$ with $f^{-1}$, and then we give the color of each pixel $Y$ of image $\mathcal{A}$ to each pixel of $P_{Y}$ in image $\mathcal{B}$ (see Fig. 3 for an illustration in 2-D). In both algorithms, some elements cannot be computed in arbitrary dimension $n$. Indeed, even if there exist algorithms to compute the Hermite Normal Form of an arbitrary square integer matrix [10, there is no generic algorithm to obtain the minimal periodicities $\left\{\alpha_{i}\right\}$ (see discussion in Sect. 5). In the next section, we focus on the minimal periodicity computation in dimension 3. 

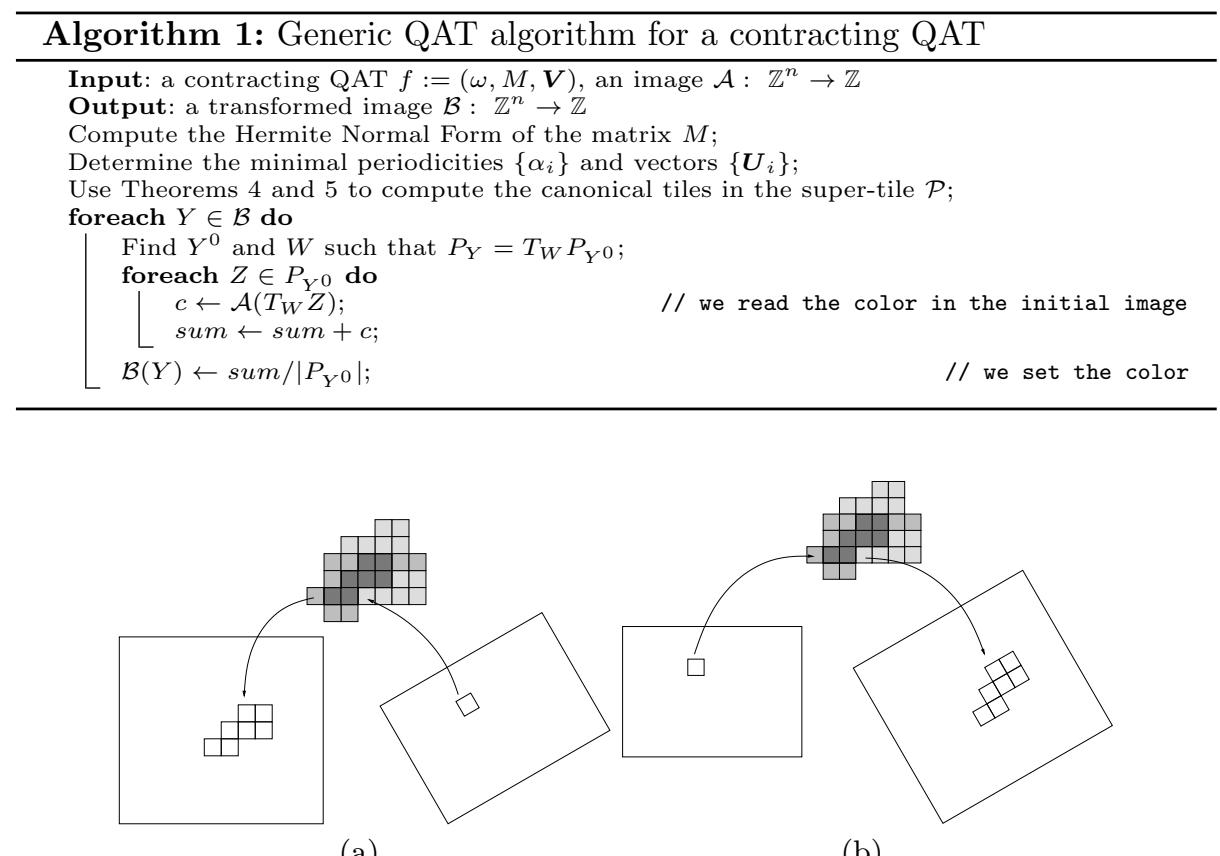

(a)

(b)

Fig. 3. Illustration in dimension 2 of the QAT algorithm when $f$ is contracting $(a)$ and dilating $(b)$. In both cases, we use the canonical tiles contained in the super-tile to speed-up the transformation.

\section{QAT in Dimension 3}

In dimension 3, we consider the following framework: we first define the Hermite Normal Form, the minimal periods and then we detail the transformation algorithm.

\subsection{Hermite Normal Form and Tile Construction}

Let us consider a $\operatorname{QAT}(\omega, M, V)$ with $M=\left(\begin{array}{lll}a_{0} & b_{0} & c_{0} \\ d_{0} & e_{0} & f_{0} \\ g_{0} & h_{0} & i_{0}\end{array}\right)$ and $V=\left(\begin{array}{l}j_{0} \\ k_{0} \\ l_{0}\end{array}\right)$.

In [7], we present explicit formulas to compute the Hermite Normal Form in 3-D. In the following, we define $H=H_{1} H_{2} H_{3} H_{4}$ and $M H=T=\left(\begin{array}{lll}a & b & c \\ 0 & d & e \\ 0 & 0 & f\end{array}\right)$.

Thanks to Hermite decomposition, we have $a>0, d>0$ and $f>0$. To construct the tile of index $(i, j, k)$ and thanks to Theorem 5, we have:

$$
A_{2}=-\left[\frac{-\omega k+l_{0}}{f}\right], B_{2}=-\left[\frac{-\omega(k+1)+l_{0}}{f}\right]
$$




$$
\begin{gathered}
A_{1}(z)=-\left[\frac{-\omega j+k_{0}+e z}{d}\right], B_{1}(z)=-\left[\frac{-\omega(j+1)+k_{0}+e z}{d}\right] \\
A_{0}(y, z)=-\left[\frac{-\omega i+j_{0}+b y+c z}{a}\right], B_{0}(y, z)=-\left[\frac{-\omega(i+1)+j_{0}+b y+c z}{a}\right]
\end{gathered}
$$

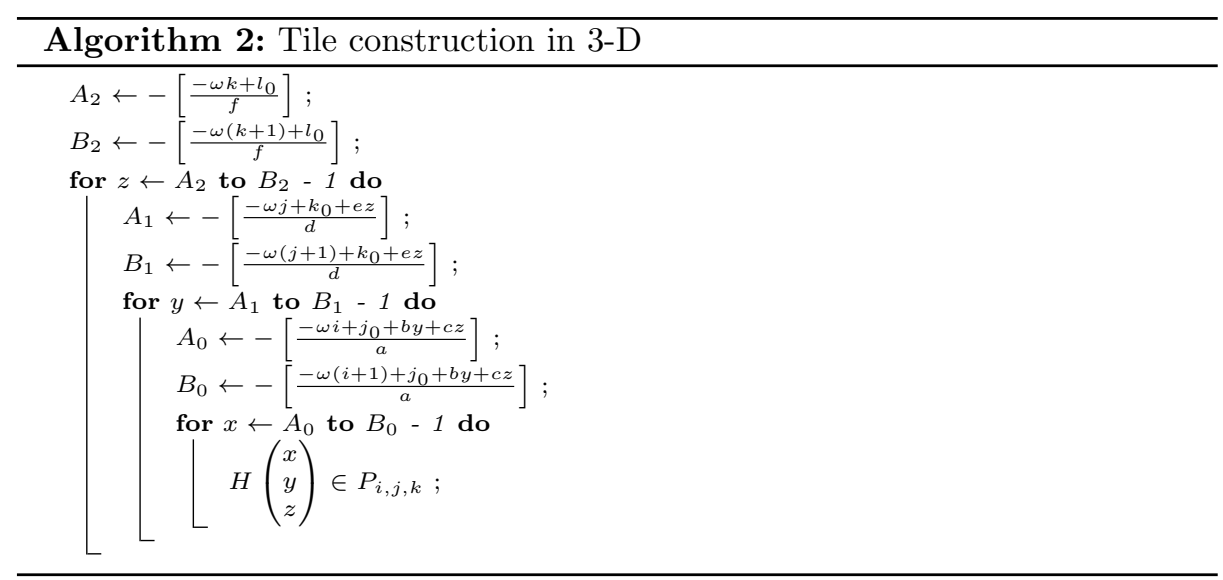

\subsection{Minimal Periodicity and Super-tile Construction}

In dimension 3 , we need to compute the periodicity along each dimension. Let us first denote $a_{h}^{\prime}=\frac{a}{\operatorname{gcd}(a, \omega)}, \omega_{h}^{\prime}=\frac{\omega}{\operatorname{gcd}(a, \omega)}, Y=\left(\begin{array}{c}\omega_{h}^{\prime} \\ 0 \\ 0\end{array}\right)$

Theorem 6 (Horizontal Periodicity). Let $\alpha_{h}=a_{h}^{\prime}$ and $U=H Y$. Then $\alpha_{h}>0, P_{i+\alpha_{h}, j, k} \equiv P_{i, j, k}$ and $\forall(i, j, k) \in \mathbb{Z}^{3}, P_{i+\alpha_{h}, j, k}=T_{U} P_{i, j, k}$.

Proof. The proof is detailed in [7].

Theorem 7. The period $\alpha_{h}$ is a minimal horizontal period, i.e. $\alpha_{h}=\alpha_{0}$.

Proof. The proof is given in [7].

Concerning the vertical period, let:

$d_{v}^{\prime}=\frac{d}{\operatorname{gcd}(d, \omega)}, \omega_{v}^{\prime}=\frac{\omega}{\operatorname{gcd}(d, \omega)}, a_{v}^{\prime}=\frac{a}{\operatorname{gcd}\left(a, b \omega_{v}^{\prime}, \omega\right)}, \phi=\frac{b \omega_{v}^{\prime}}{\operatorname{gcd}\left(a, b \omega_{v}^{\prime}, \omega\right)}, \omega_{v}^{\prime \prime}=\frac{\omega}{\operatorname{gcd}\left(a, b \omega_{v}^{\prime}, \omega\right)}$, $\alpha_{v}^{\prime}=\operatorname{gcd}\left(a_{v}^{\prime}, \omega_{v}^{\prime \prime}\right), u_{1}$ and $v_{1}$ are such that $: a_{v}^{\prime} u_{1}+\omega_{v}^{\prime \prime} v_{1}=\operatorname{gcd}\left(a_{v}^{\prime}, \omega_{v}^{\prime \prime}\right)\left(=\alpha_{v}^{\prime}\right)$, $\beta_{0}=-\phi v_{1}, Y=\left(\begin{array}{c}-\phi u_{1} \\ \omega_{v}^{\prime} \alpha_{v}^{\prime} \\ 0\end{array}\right)$ 
Theorem 8 (Vertical Periodicity). Let $\alpha_{v}=d_{v}^{\prime} \alpha_{v}^{\prime}, U=H Y$. Then $\alpha_{v}>$ $0, P_{i, j+\alpha_{v}, k} \equiv P_{i+\beta_{0}, j, k}$ and $\forall(i, j, k) \in \mathbb{Z}^{3}, P_{i, j+\alpha_{v}, k}=T_{U} P_{i+\beta_{0}, j, k}$.

Proof. The proof is given in [7].

Theorem 9. The period $\alpha_{v}$ is a minimal vertical period, i.e. $\alpha_{v}=\alpha_{1}$.

Proof. The proof is detailled in 7 .

For the last period, let us consider:

$f_{d}^{\prime}=\frac{f}{\operatorname{gcd}(\omega, f)}, \omega_{d}^{\prime}=\frac{\omega}{\operatorname{gcd}(\omega, f)}, d_{d}^{\prime}=\frac{d}{\operatorname{gcd}\left(d, e \omega_{d}^{\prime}, \omega\right)}, \phi=\frac{e \omega_{d}^{\prime}}{\operatorname{gcd}\left(d, e \omega_{d}^{\prime}, \omega\right)}, \omega_{d}^{\prime \prime}=\frac{\omega}{\operatorname{gcd}\left(d, e \omega_{d}^{\prime}, \omega\right)}$, $u_{1}$ and $v_{1}$ are such that $: d_{d}^{\prime} u_{1}+\omega_{d}^{\prime \prime} v_{1}=\operatorname{gcd}\left(d_{d}^{\prime}, \omega_{d}^{\prime \prime}\right), \psi=c \omega_{d}^{\prime} \operatorname{gcd}\left(d_{d}^{\prime}, \omega_{d}^{\prime \prime}\right)-b \phi u_{1}$,

$a_{d}^{\prime}=\frac{a}{\operatorname{gcd}\left(a, \psi, \omega, \frac{\omega_{d}^{\prime \prime} b}{\operatorname{gcd}\left(d_{d}^{\prime}, \omega_{d}^{\prime \prime}\right)}\right)}, \psi^{\prime}=\frac{\psi}{\operatorname{gcd}\left(a, \psi, \omega, \frac{\omega_{d}^{\prime \prime} b}{\operatorname{gcd}\left(d_{d}^{\prime}, \omega_{d}^{\prime \prime}\right)}\right)}$,

$\omega_{d}^{\prime \prime \prime}=\frac{\omega}{\operatorname{gcd}\left(a, \psi, \omega, \frac{\omega_{d}^{\prime \prime} b}{\operatorname{gcd}\left(d_{d}^{\prime}, \omega_{d}^{\prime \prime}\right)}\right)}, \chi=\frac{\frac{\omega_{d}^{\prime \prime} b}{\operatorname{gcd}\left(d_{d}^{\prime}, \omega_{d}^{\prime \prime}\right)}}{\operatorname{gcd}\left(a, \psi, \omega, \frac{\omega_{d}^{\prime \prime} b}{\operatorname{gcd}\left(d_{d}^{\prime}, \omega_{d}^{\prime \prime}\right)}\right)}$,

$\alpha_{d}^{\prime \prime}=\operatorname{gcd}\left(a_{d}^{\prime}, \chi, \omega_{d}^{\prime \prime \prime}\right), \alpha_{d}^{\prime}=\alpha_{d}^{\prime \prime} \operatorname{gcd}\left(d_{d}^{\prime}, \omega_{d}^{\prime \prime}\right)$

$u_{2}, v_{2}$ and $w_{2}$ are such that $: a_{d}^{\prime} u_{2}+\chi v_{2}+\omega_{d}^{\prime \prime \prime} w_{2}=\operatorname{gcd}\left(a_{d}^{\prime}, \chi, \omega_{d}^{\prime \prime \prime}\right)\left(=\alpha_{d}^{\prime \prime}\right)$,

$k=-\psi^{\prime} v_{2}, \beta_{0}=-\psi^{\prime} w_{2}, \beta_{1}=-\phi v_{1} \alpha_{d}^{\prime \prime}-k \frac{d_{d}^{\prime}}{\operatorname{gcd}\left(d_{d}^{\prime}, \omega_{d}^{\prime \prime}\right)}, Y=\left(\begin{array}{c}-\psi^{\prime} u_{2} \\ -\phi u_{1} \alpha_{d}^{\prime \prime}+k \frac{\omega_{d}^{\prime \prime}}{\operatorname{gcd}\left(d_{d}^{\prime}, \omega_{d}^{\prime \prime}\right)} \\ \alpha_{d}^{\prime} \omega_{d}^{\prime}\end{array}\right)$

Theorem 10 (Depth Periodicity). Let $\alpha_{d}=\alpha_{d}^{\prime} f_{d}^{\prime}, U=H Y$. Then

$\alpha_{d}>0 \quad P_{i, j, k+\alpha_{d}} \equiv P_{i+\beta_{0}, j+\beta_{1}, k} \quad$ and $\forall(i, j, k) \in \mathbb{Z}^{3}, P_{i, j, k+\alpha_{d}}=T_{U} P_{i+\beta_{0}, j+\beta_{1}, k}$

Proof. The proof is detailed in [7].

Theorem 11. The period $\alpha_{d}$ is a minimal depth period, i.e. $\alpha_{d}=\alpha_{2}$.

Proof. The proof is detailed in [7.

Based on these periods, we can construct the super-tile and all the initial period tiles. To design a transformation algorithm, for each point $X \in \mathcal{P}$, we need to determine the tile index $Y$ to which $X$ belongs to. Since $X \in P_{Y} \Leftrightarrow\left[\frac{M X+V}{\omega}\right]=$ $Y$, Algorithm 3 details the initial period tile construction with scanning points in $\mathcal{P}$. The computational cost of Alg. 3 exactly corresponds to the number of tiles in the initial period.

Proposition 2. The number of tiles of the initial period is $\omega_{d}^{\prime} \omega_{d}^{\prime \prime} \omega_{d}^{\prime \prime \prime}$.

In the Proposition statement, we do not give the closed formula. However, $\omega_{d}^{\prime} \omega_{d}^{\prime \prime} \omega_{d}^{\prime \prime \prime}$ is equal to $\omega^{3}$ divided by a product of three $\operatorname{gcd}()$. 


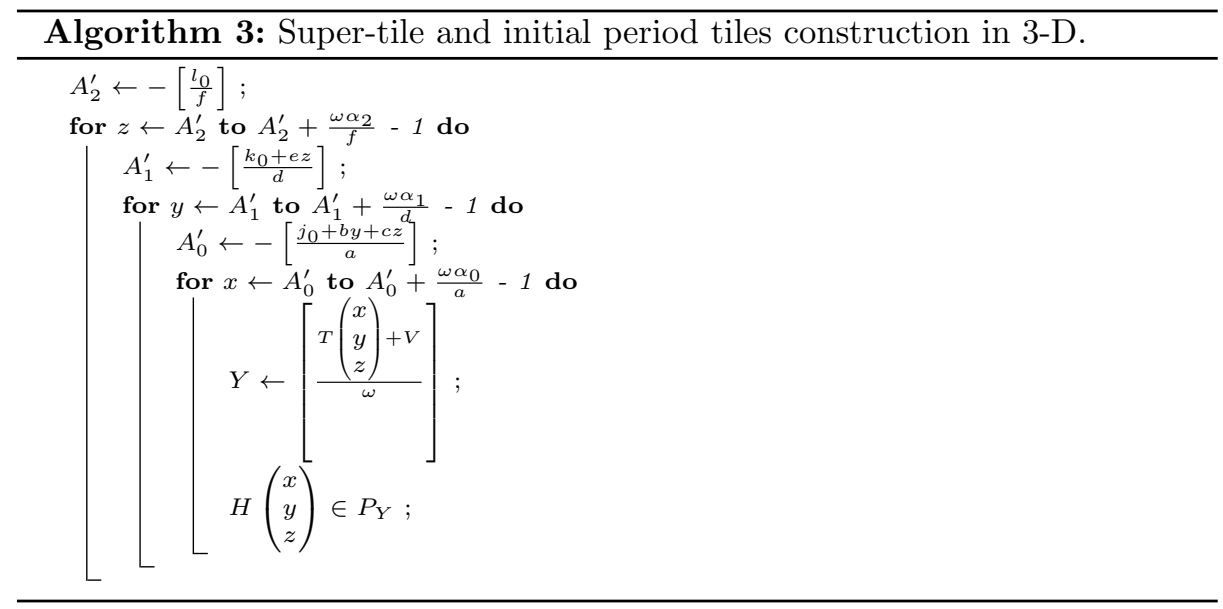

Proof. The proof is detailed in [7].

Using Theorems 4 and 5 we have

$$
\theta_{0}=\frac{\operatorname{lcm}\left(\alpha_{0}, \alpha_{1}, \alpha_{2}\right)}{\alpha_{0}}, \theta_{1}=\frac{\operatorname{lcm}\left(\alpha_{0}, \alpha_{1}, \alpha_{2}\right)}{\alpha_{1}}, \theta_{1}=\frac{\operatorname{lcm}\left(\alpha_{0}, \alpha_{1}, \alpha_{2}\right)}{\alpha_{2}},
$$

and

$\mathcal{P}=\left\{H\left(\begin{array}{l}x \\ y \\ z\end{array}\right) / A_{2}^{\prime} \leq z<B_{2}^{\prime}, A_{1}^{\prime}(z) \leq y<B_{1}^{\prime}(z)\right.$ and $\left.A_{0}^{\prime}(y, z) \leq x<B_{0}^{\prime}(y, z)\right\}$,

with $A_{2}^{\prime}=-\left[\frac{l_{0}}{f}\right], A_{1}^{\prime}(z)=-\left[\frac{k_{0}+e z}{d}\right], A_{0}^{\prime}(y, z)=-\left[\frac{j_{0}+b y+c z}{a}\right], B_{2}^{\prime}=A_{2}^{\prime}+\frac{\omega \alpha_{2}}{f}$, $B_{1}^{\prime}(z)=A_{1}^{\prime}(z)+\frac{\omega \alpha_{1}}{d}$, and $B_{0}^{\prime}(y, z)=A_{0}^{\prime}(y, z)+\frac{\omega \alpha_{0}}{a}$ (see [7 for details).

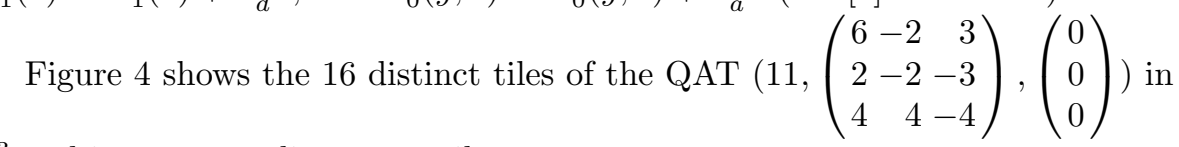
$\mathbb{Z}^{3}$ and its correponding super-tile.

\subsection{QAT Algorithm in 3-D}

To obtain the overall QAT algorithm, we need to find both the initial period tile index and the translation vector associated to a given tile $P_{i, j, k}$. Hence, thanks to Theorem 3, we have

$$
\begin{aligned}
& \forall(i, j, k) \in \mathbb{Z}^{3}, P_{i, j, k}=T_{W} P_{i^{0}, j^{0}, k^{0}} \text { with } W=w_{0} U_{0}+w_{1} U_{1}+w_{2} U_{2} \\
& \text { and } 0 \leq k^{0}=\left\{\frac{k}{\alpha_{2}}\right\}<\alpha_{2}, w_{2}=\left[\frac{k}{\alpha_{2}}\right], \quad 0 \leq j^{0}=\left\{\frac{j+w_{2} \beta_{1}^{2}}{\alpha_{1}}\right\}<\alpha_{1}, w_{1}=\left[\frac{j+w_{2} \beta_{1}^{2}}{\alpha_{1}}\right], \\
& 0 \leq i^{0}=\left\{\frac{i+w_{1} \beta_{0}^{1}+w_{2} \beta_{0}^{2}}{\alpha_{0}}\right\}<\alpha_{0}, w_{0}=\left[\frac{i+w_{1} \beta_{0}^{1}+w_{2} \beta_{0}^{2}}{\alpha_{0}}\right] .
\end{aligned}
$$




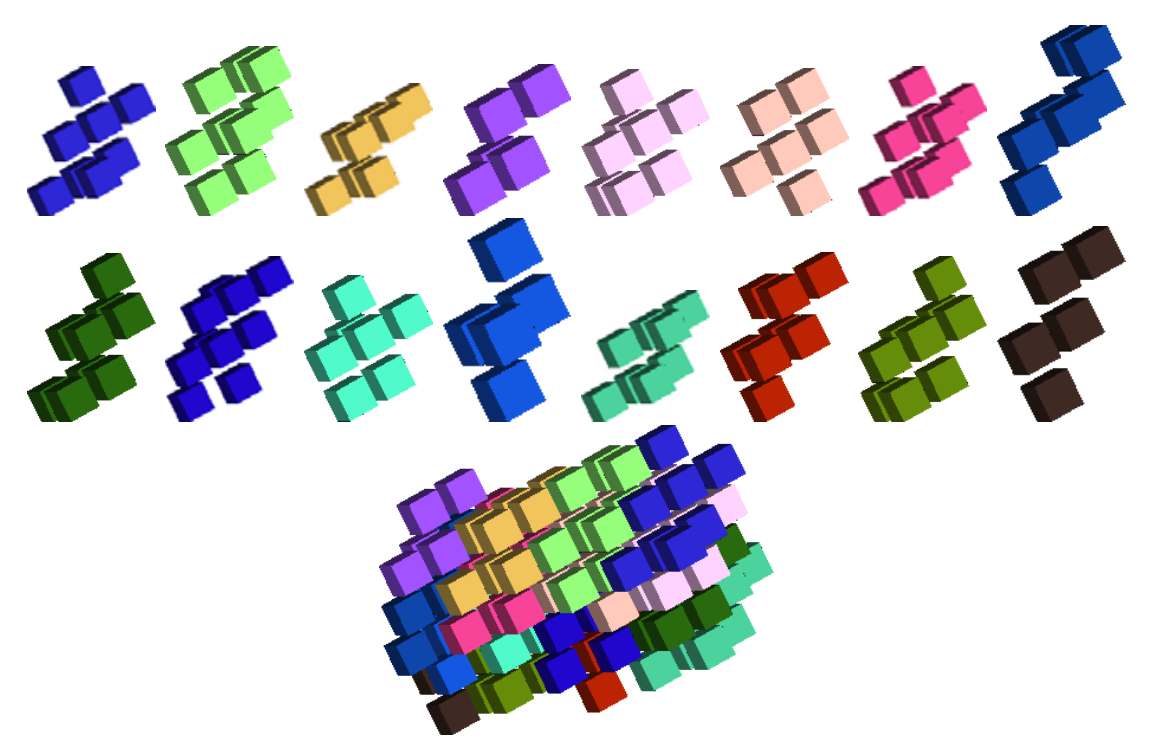

Fig. 4. All distinct tiles of a QAT in $\mathbb{Z}^{3}$ and its super-tile.

\section{Experiments}

The algorithms were implemented in both $2 \mathrm{D}$ and 3D, with different refinements in order to be able to compare the implementations. For details on the 2-D algorithms, refer to [7]. The backward mapping (B. M. for short) implementation let

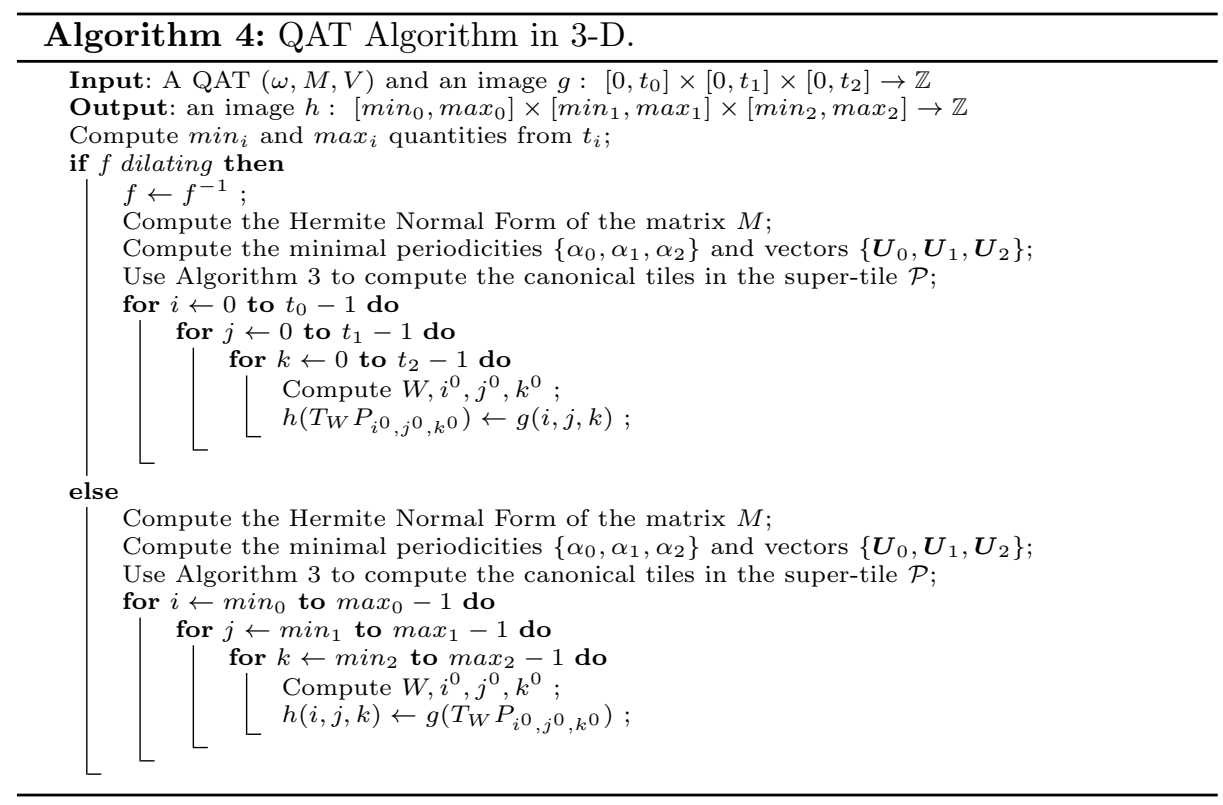




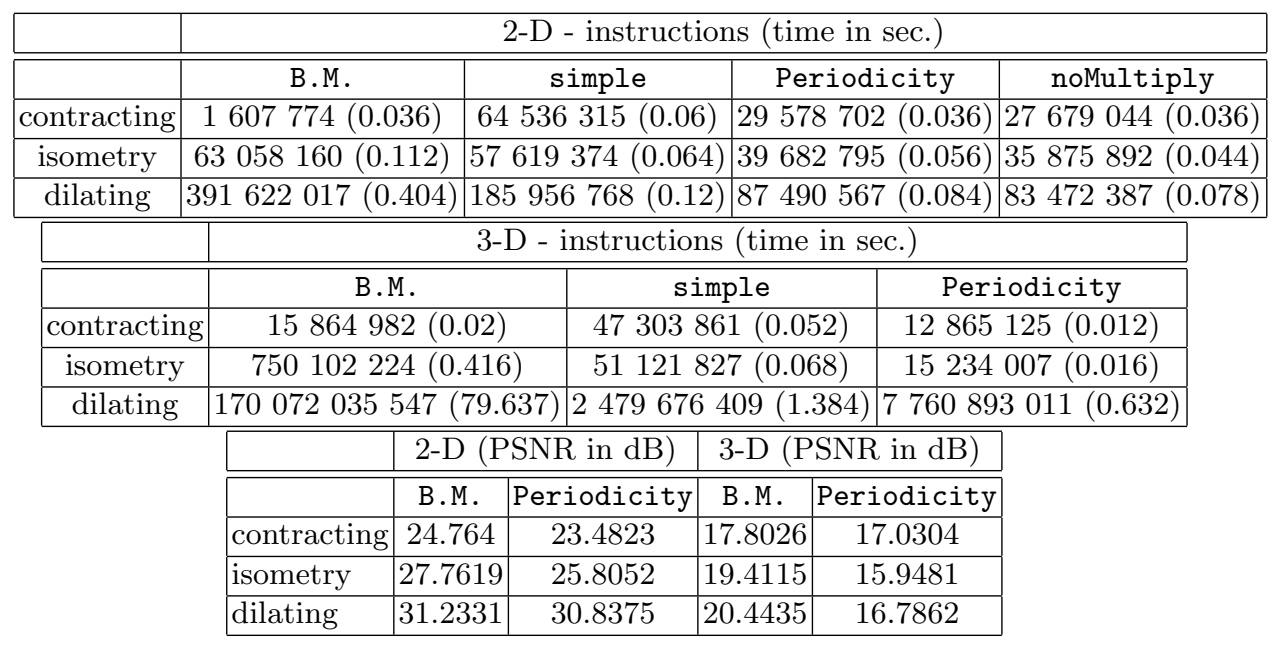

Table 1. Comparative evaluation in $2-\mathrm{D}$ and $3-\mathrm{D}$. The last table presents the PSNR evaluation of the composition $f \cdot f^{-1}$.

us compare the tile periodicity method with the widely used backward mapping method 9 . The simple implementation does not use tiles periodicity and uses algorithm 2 for each tile. The periodicity implementation uses the periodicity and the algorithm 4. The noMultiply implementation additionally uses a method presented in 4 which uses a handling of remains instead of computing a matrix product in 3 . The experiments are performed on an Intel(C) Centrino (C) Duo T2080 ( $2 \times 1.73 \mathrm{GHz})$ in monothread and we give on one hand the time of computation and on the other hand the number of elementary instructions. The QATs used are the following : In 2-D:

$$
\left(\omega,\left(\begin{array}{cc}
3 & -4 \\
4 & 3
\end{array}\right),\left(\begin{array}{l}
0 \\
0
\end{array}\right)\right) \text { where } \omega=\left\{\begin{array}{l}
10 \text { for the contracting application } \\
5 \text { for the isometry } \\
2 \text { for the dilating application }
\end{array}\right.
$$

In 3-D:

$$
\left(\omega,\left(\begin{array}{ccc}
9 & -20 & -12 \\
12 & 15 & -16 \\
20 & 0 & 15
\end{array}\right),\left(\begin{array}{l}
0 \\
0 \\
0
\end{array}\right)\right) \text { where } \omega=\left\{\begin{array}{l}
100 \text { for the contracting application } \\
25 \text { for the isometry } \\
4 \text { for the dilating application }
\end{array}\right.
$$

The pictures are of size : $200 \times 171$ in 2-D and $10 \times 10 \times 10$ in 3-D (simple cube).

Figure 5 illustrates the results in dimension 2. As expected, when comparing B.M. and Periodicity, results are similar for both contracting and isometry QATs. Differences appear when dilating QAT is considered. Indeed, since a unique color is associated to a tile in the Periodicity algorithm, the transformed image contains sharp edges (Fig $5-(l)$ ) On the other hand, the interpolation process in the B.M. algorithm makes the image blurred. To compare the 


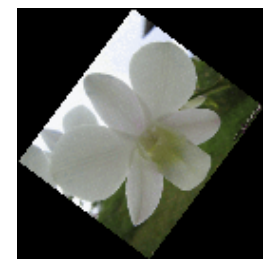

(a)

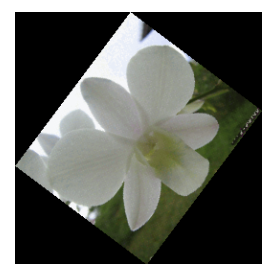

(e)

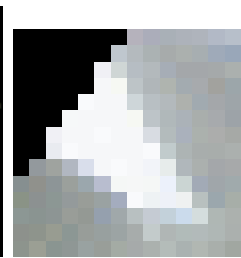

(b)

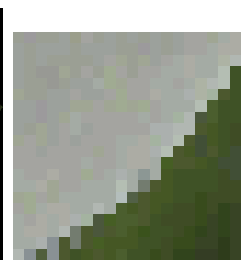

(f)

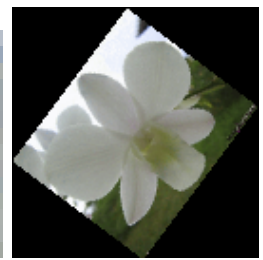

(c)

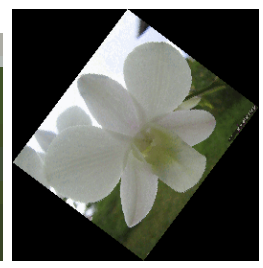

(g)

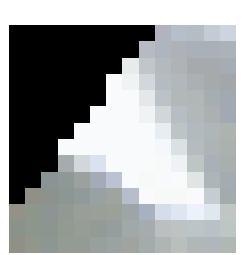

(d)

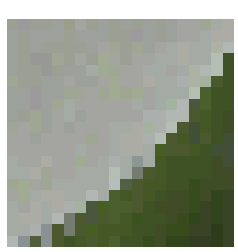

(h)

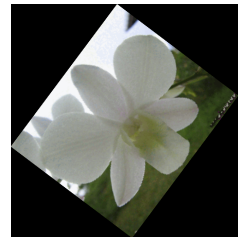

(i)

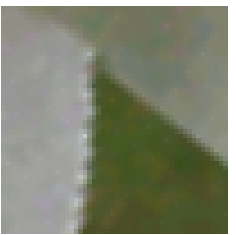

(j)

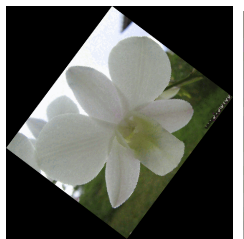

(k)

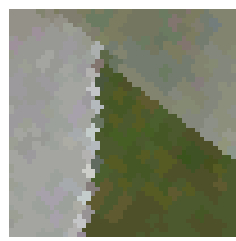

(l)

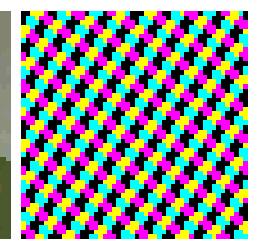

(m)

Fig. 5. Results in dimension 2: $(a-d)$ Contracting QAT (B.M. $(a-b)$ and Periodicity $(c-d)) ;(e-h)$ Isometry (B.M. and Periodicity); $(i-l)$ Dilating (B.M. and Periodicity). $(m)$ illustrates the tile structure of the dilating QAT on the same square as in $(l)$.

time efficiency (Table 1), we have considered two quantities: the total number of elementary operations of the main loof $\mathrm{f}^{5}$ and the overall computational time in seconds. Table 1 and Figure 6 present the results in dimension 3. For the sake of clarity, we have only considered an input binary image but the transformation algorithms can be applied to 3-D color images. We have also performed a peak signal-to-noise ratio (PSNR for short, given in decibel dB) computation between the input image in 2-D and 3-D, and the result of the composition $f \cdot f^{-1}$. This test has been designed to evaluate the propagation of the error through the transformations with a signal processing tool. As presented in Table 1, the distortion induced by the proposed method is always smaller than the one induced by the backward mapping technique. Note that to have relevant measurements, we have used a density 3 -D volume for the 3-D test (see Fig. 6. $(h)$ ).

\footnotetext{
${ }^{5}$ obtained with the valgrind profiling tool.
} 


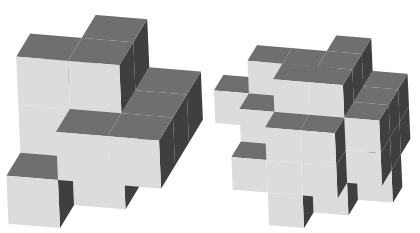

(a) (b)

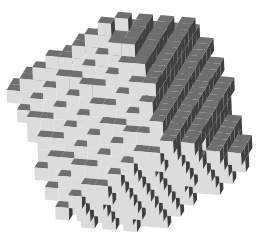

(c)

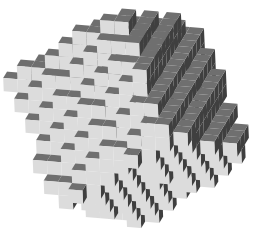

(d)

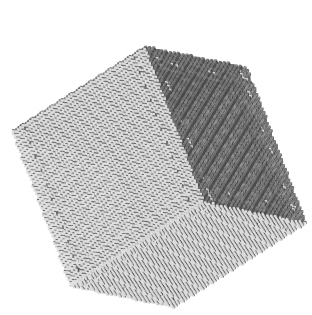

(e)

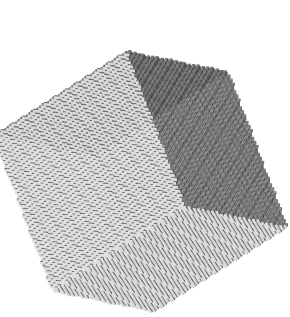

(f)

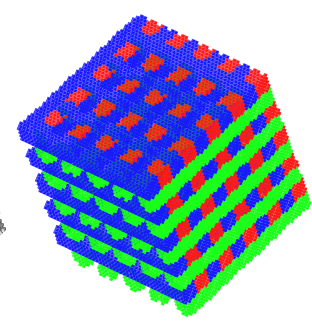

(g)

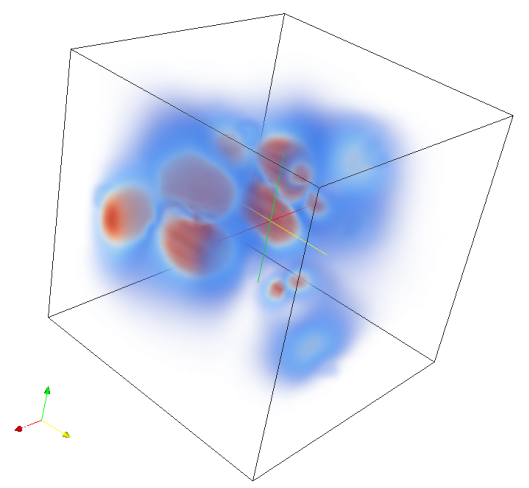

(h)

Fig. 6. Results in dimension 3: $(a-b)$ Contracting (B.M. and Periodicity), $(c-d)$ Isometry and $(e-f)$ Dilating (B.M. and Periodicity). ( $g$ ) illustrates the tile structure of the dilating QAT. $(h)$ is the $32 \times 32 \times 32$ density volume used in the $f \cdot f^{-1}$ composition test (from the TC18 data-set http://www.tc18.org).

\section{Conclusion and Future Works}

In this paper, we have first re-demonstrated an existing result in dimension 2 with our formalism and provide a generalization in dimension 3 of fast image transformations with QAT. Compared to a classical image transformation technique, we have also illustrated the strength of such arithmetical methods to speed up transformations in higher dimensions. As detailed above and based on the generic $n-D$ transformation algorithm proposed in [6/7/8, we had to 
perform specific computation to obtain the minimal periods in dimension 2 and 3. A very challenging future work is to define a framework to compute these minimal periods when a specific dimension is considered. In order to achieve this goal and instead of having explicit formulas, we plan to investigate an algorithmic solution based on the $n-D$ canonical tile counting algorithm proposed in 6]. Furthermore, in dimension 2 and 3, comparisons to other transformation algorithms, such as Fourier based technique, are also of interest.

\section{References}

1. Nehlig, P., Ghazanfarpour, D.: Affine Texture Mapping and Antialiasing Using Integer Arithmetic. Computer Graphics Forum 11(3) (1992) 227-236

2. Jacob, M.: Transformation of digital images by discrete affine applications. Computers \& Graphics 19(3) (1995) 373-389

3. Nehlig, P.: Applications quasi affines: pavages par images réciproques. Theoretical Computer Science 156(1-2) (1996) 1-38

4. Jacob, M.: Applications quasi-affines. PhD thesis, Université Louis Pasteur, Strasbourg, France (1993)

5. Jacob-Da Col, M.: Applications quasi-affines et pavages du plan discret. Theoretical Computer Science 259(1-2) (2001) 245-269 english version: http://dpt-info. u-strasbg.fr/ jacob/articles/paving.pdf.

6. Jacob-Da Col, M.A., Tellier, P.: Quasi-linear transformations and discrete tilings. Theoretical Computer Science 410(21-23) (2009) 2126 - 2134

7. Blot, V., Coeurjolly, D.: Quasi-affine transform in higher dimension. Technical Report RR-LIRIS-2009-010, Laboratoire LIRIS (April 2009) http://liris.cnrs. fr/publis?id=3853

8. Blot, V., Coeurjolly, D.: Quasi-affine transform in higher dimension. In: 15th International Conference on Discrete Geometry for Computer Imagery. LNCS, SpringerVerlag (submitted).

9. Foley, J.D., van Dam, A., Feiner, S.K., Hughes, J.F.: Computer Graphics: Principles and Practice. second edn. Addison-Wesley, Reading, Mass. (1990)

10. Storjohann, A., Labahn, G.: Asymptotically fast computation of hermite normal forms of integer matrices. In: ISSAC '96: Proceedings of the 1996 international symposium on Symbolic and algebraic computation, New York, NY, USA, ACM (1996) 259-266 